\title{
A BRIEF OVERVIEW ON DIFFERENT ANIMAL DETECTION METHODS
}

\author{
Prof. Sachin Sharma ${ }^{1}$ and Dr. D. J. Shah ${ }^{2}$ \\ ${ }^{1}$ Department of Electronics \& Communication, GTU, Ahmedabad, India \\ sharma.f@gmail.com \\ ${ }^{2}$ Department of Electronics \& Communication, GTU, Ahmedabad, India \\ djshah99@gmail.com
}

\begin{abstract}
Researches based on animal detection plays a very vital role in many real life applications. Applications which are very important are preventing animal vehicle collision on roads, preventing dangerous animal intrusion in residential area, knowing locomotive behavioural of targeted animal and many more. There are limited areas of research related to animal detection. In this paper we will discuss some of these areas for detection of animals.
\end{abstract}

\section{KEYWORDS}

Animal Detection, Radio-Frequency Identification (RFID), Global Positioning System (GPS), Image Processing, Threshold, Segmentation

\section{INTRODUCTION}

Animal detection is a very important and emerging area due to a large number of real life applications. Various animal detection methods and warning systems are used for indicating the presence of animals on the roads or residential area. Applications which are very important in real life are preventing animal vehicle collision on roads, preventing dangerous animal intrusion in residential area, knowing locomotive behavioural of targeted animal etc. All these applications can be narrowed down to three areas namely detection, tracking and identification of animals.

The very first area that is detection of animals is applied in various fields of real life applications. As an example hundreds of camel-vehicle accidents were reported every year causing numerous deaths and loss of property running into millions of Saudi Riyals. To address this problem, a deployable and intelligent Camel Vehicle Accident Avoidance System (CVAAS) was designed using global positioning system technology [1]. Researchers in [2] developed an algorithm for light detection and ranging (LIDAR) data to enable fisherman to find the right location of fishes in deep sea. For maintaining human safety and security by detecting possible dangerous animal intrusions into the residential area, researchers in [3] used microDoppler signals.

DOI : 10.5121/sipij.2013.4307 
Signal \& Image Processing : An International Journal (SIPIJ) Vol.4, No.3, June 2013

The second area that is tracking of animals is important for monitoring or observing the locomotive behavior of animals and its surroundings. Researchers in [4] developed zoological systems for tracing an animal, identification, and anti-theft for the management and security of animal in zoo with the help of sensor, radio-frequency identification (RFID), and global positioning system (GPS). By tracking and observing the animal movements, it helps us to have a better understanding on how an animal behaves and interacts with its environment.

The third area that is identification of animals is very important in identifying the targeted animal and its behavior. Identification of animals helps human being to monitor and manage animals easier. Researchers in [5] designed and developed RFID-based mobile monitoring system for better management of animals in dynamic information retrieving, location tracking and to help users over a wireless network.

This paper mainly focuses on various animal detection methods. There is a review limited to the methods that use images or video which is given in the next section.

\section{Animal Detection Methods in IMAge \& Video Processing}

Researches based on animal detection plays a very vital role in many real life applications. Applications which are very important are preventing animal vehicle collision on roads, preventing dangerous animal intrusion in residential area, knowing locomotive behavioural of targeted animal and many more.

\subsection{Human Prediction approach for animal detection}

Initial researches on animal detection are based on to observe how fast and accurate human eyes can detect the presence of animals in original image. This approach is very good and reliable if the animal detection distance is near and doesn't have lighting problems. This method for animal detection by human eyes is also reliable if seen from the computational point of view. Work done in [6] showed that a human observer is able to make a decision whether a momentarily flashed animal image is having the presence of an animal as fast as $150 \mathrm{~ms}$.

Even though this approach of human prediction for animal detection is effective and achieves some reasonable result or level, human eyes do have some serious limitations. Human eyes can get tired or exhausted easily causing a limitation in the effectiveness and accuracy of the method (algorithm). Human eyes need some rest and can't work efficiently for 24 hours a day to perform animal detection. These limitations can be restricted by using computer vision in image processing for animal detection.

\subsection{Threshold Segmentation approach for animal detection}

For extracting the targeted animal's details from background, this approach can be used. The basic idea of this approach is simple in which the pixels in the image having intensities or values greater than the threshold are set to white (i.e. intensity 255) and those pixels having intensities or values less than the threshold value are set to black (i.e. intensity 0 ). There are different types of thresholding like adaptive thresholding or dynamic thresholding and optimal thresholding which are very important topics image processing but in this paper we will restrict to simple concept of thresholding only. 
Signal \& Image Processing : An International Journal (SIPIJ) Vol.4, No.3, June 2013

In [7], the object or animal is found by using background subtraction method after getting the background image. Work done in [8] shows that it is very difficult and tedious to choose the threshold value as the background image changes periodically.

\subsection{Power Spectrum approach for animal detection}

Researchers have tried to find out whether the presence of animal in the scene or image will affect the power spectral of the image or not which can be defined as the amplitude of the signal in the frequency domain.

The power spectrum can be constructed by transforming images from $\mathrm{s}$ (spatial) domain to frequency domain with the help of the transformation function like Fourier transform. Work carried out in [9] shows that this approach is not suitable if a person wants quick result or wants to detect the animals very quickly as this approach takes more time.

\subsection{Face Detection approach for animal detection}

For monitoring or observing the locomotive behavior of animals and their interaction with the surroundings, researchers in [10] applied detection and tracking of targeted animal faces using Haar-like feature and Adaboost classifiers. When it is positive that targeted animal has been detected, video recorders turned on to extend battery life time and to ensure that recorded video contains a correct research value.

This method is very crucial and important in situation whereby video person is not suitable to present at the recording scene for safety issue or video person might be scared off some timid animal away. The measurement of animal faces is done by utilizing face detection method with different local contrast configuration of luminescence channel to detect the image region of animal faces.

\section{SUMMARY \& FUTURE WORK}

There are lots of issues that need to be taken into account for developing an efficient animal detection algorithm. Animals come from nowhere so you can't predict their presence and also the speed of the animals can't be monitored or detected. There is the lighting problem also, wherein a sudden change of lighting effect can affect the effectiveness in detecting the presence of animal intrusion. Also each animal has its own characteristics and behavior with the surroundings which leads to a problem in identification of correct animals. Apart from that moving background, such as trees or leaves caused by wind might be regarded as foreground image and some stationary animal which remain still for a long time can be mistakenly interpreted as background image by the algorithms. So in future lots of work and researches needs to be done in developing an effective animal detection algorithm

\section{ACKNOWLEDGEMENTS}

The authors would like to thank everyone, just everyone! 
Signal \& Image Processing : An International Journal (SIPIJ) Vol.4, No.3, June 2013

\section{REFERENCES}

[1] M.S. Zahrani, Khaled Ragab and Asrar Ul Haque, "Design of GPS based system to avoid camelvehicle collisions: A Review," Asian Journal of Applied Sciences 4 (4): 362 - 377, 2011.

[2] V. Mitra, C. Jiu Wang, and G. Edwards, "Neural network for LIDAR detection of fish," in Neural Networks Proceedings of the International Joint Conference, vol. 2, pp. 1001-1006, 2003.

[3] D. Tahmoush and J. Silvious, "Modeled gait variations in human micro-Doppler," 2010 11th International Radar Symposium (IRS), pp. 1-4, 2010.

[4] S. H. Kim, D. H. Kim, and H. D. Park, "Animal situation tracking service using RFID, GPS, and sensors," 2010 Second International Conference on Computer and Network Technology (ICCNT), pp. 153-156, 2010.

[5] J. S. L. Ting, S. K. Kwok, W. B. Lee, H. C. A. Tsang, and B. C. F. Cheung, "A dynamic RFID-based mobile monitoring system in animal care management over a wireless network," Wireless Communications Networking and Mobile Computing, vol. 2, pp. 2085-2088, 2007.

[6] M. F. Thorpe, A. Delorme, and S. T. C. Marlot, "A limit to the speed processing in ultra-rapid visual categorization of novel natural scene," Cognitive Neuroscience, pp. 171-180, 2003.

[7] C. Peijiang, "Moving object detection based on background extraction," Computer Network and Multimedia Technology (CNMT), 2009.

[8] J. C. Nascimento and J. S. Marques, "Performance evaluation of object detection algorithms for video surveillance," IEEE Transactions on Multimedia, vol. 8, pp. 761-774, 2006.

[9] F. A. Wichmann, J. Drewes, P. Rosas, and K. R. Gegenfurtner, "Animal detection in natural scenes: Critical review revisited," Journal of Vision, vol. 10, no. 4, pp. 1-27, 2010.

[10] T. Burghardt and J. Calic, "Real-time face detection and tracking of animals," 8th Seminar on Neural Network Applications in Electrical Engineering, pp. 27-32, 2006. 
Signal \& Image Processing : An International Journal (SIPIJ) Vol.4, No.3, June 2013

\section{Authors:}

Sachin Sharma, Ph.D. pursuing, is Assistant Professor, Electronics and Communication Department, SVBIT, Gandhinagar (Gujarat). He is having more than 5 years of experience in Academics, Research \& Industry. He has written books on ABS Implementation using Fuzzy Logic based Controller and Fundamentals of Image Processing. He has also published numerous articles related to Image Processing, Digital Signal Processing, and Intelligent Transportation Systems. He is an active member of several professional societies, including ISTE, IEEE and SAE.

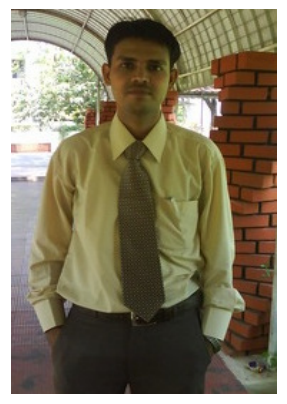

Dr. Dharmesh Shah is working as Principal at SPCE, Visnagar (Gujarat). He is also the Dean Engineering (Zone II), GTU, Ahmedabad. He is having more than 15 years of experience in Academics, Research \& Industry. He has written a book on Fundamentals of Image Processing for the final year students. He has also published numerous articles related to VLSI, Digital Signal Processing, and Image Processing. He is an active member of several professional societies, including IETE, ISTE and IEEE. 